\title{
Realization of All Optical Full Adder by Utilizing DM Soliton Pulses
}

\author{
Ank Raj, M.E student \\ Department of Electronics \& \\ Communication Engineering \\ PEC University of Technology, \\ Sector-12 Chandigarh, India
}

\author{
Kadam Bhambri, \\ Research Scholar \\ Department of Electronics \& \\ Communication Engineering \\ PEC University of Technology, \\ Sector-12 Chandigarh, India
}

\author{
Neena Gupta, Professor \\ Department of Electronics \& \\ Communication Engineering \\ PEC University of Technology, \\ Sector-12 Chandigarh, India
}

\begin{abstract}
Requirement of high bit rate circuits is increasing day by day, for achieving this we can use all optical processing digital circuits. These circuits are cost efficient and having high speed processing. The basic component for all-optical processing is optical transistor, which can be designed using all-optical logic gates. Many higher level circuits such as adders, Flip-flops, counters can be designed by utilizing these logic gates. In this paper, the Full Adder is proposed which utilizes dispersion managed soliton pulses as input by cross gain modulation of Semiconductor amplifier is simulated at $100 \mathrm{~Gb} / \mathrm{s}$. The circuit employs two all-optical XOR and AND gates and one OR gate
\end{abstract}

\section{General Terms}

SOA (Semiconductor Optical Amplifier), DM (Dispersion Managed) Solitons, XGM (Cross Gain Modulation), EDFA (Erbium Doped Fiber Amplifier), GVD (Group Velocity Dispersion)

\section{Keywords}

SOA (Semiconductor Optical Amplifier), DM(Dispersion Managed) Solitons, XGM(Cross Gain Modulation), EDFA(Erbium Doped Fiber Amplifier).

\section{INTRODUCTION}

Internet as an industry is largely based around fiber due to its high switching speed so, all optical processing (AOP) is the field of teeming in research and it will be very useful in near future all-optical networks because of ultra high speed of optical circuits and speed constraints in electronic circuits. Optical networks designed for the business access environment will need to incorporate lower-cost systems to be cost effective and enable the wavelength services. Nonlinear effects are intensity dependent, so in optical fibers, these effects can be used for high-speed processing of optical signals. Also, High-speed switching is possible because of the non-resonant nature of fiber nonlinearity.

All-optical logic gates are used to replace the transistor in electrical circuits [1]. Now, some higher level digital circuits such as half-adder, full adder, flip-flop, shift registers, etc can be realized by utilizing these optical gates. This shall aid the important part in the development of all-optical processing in all-optical computers.

In all-optical computing, the three basic functions are switching, arithmetic operations and storage. Full adder is proposed for arithmetic operations which can simultaneously produce sum and carry [10].
The most widely used all-optical schemes for implementation of adder are All optical integrated full adder-subtractor and demultiplexer using SOA-based Mach-Zehnder interferometer [2], photonic crystals [3], dark/ bright solitons [4],quantum dot SOA based MZI[5]

In this paper a simple novel all-optical full adder is simulated at $100 \mathrm{~Gb} / \mathrm{s}$ by employing the XGM(Cross Gain Modulation) in semiconductor optical amplifier This circuit uses dispersion managed soliton pulses as input signal and it can be used opamp circuits, adaptive filter implementation, processors, arithmetic circuits etc.

NRZ, RZ, CS-RZ and soliton pulses are used as the input signal in most of the researches till date [7]. Optical soliton pulses assure of transmitting data at high speed in optical fibers over long distances but these pulses face difficulties such as timing jitter, Raman self frequency shift, third-order, and the linear fiber loss creep that crucially limits the available capacity and transmission distance in soliton based system and collectively distort the propagation of pulse. Fortunately, a significant breakthrough has finally come to overcome these limitations as dispersion management Dispersion managed (DM) soliton enhance energy as compared to classical soliton in fiber and allows suppression of jitter by reducing the average GVD without sacrificing of the signal-to-noise ratio [6].

In the proposed design of Full adder, sum and carry is generated using DM soliton as input pulses and exploiting nonlinear properties of SOA and XGM(Cross Gain Modulation). Cross-Phase Modulation (XPM) allows better control of switching using suitable control pulses In comparison to other approaches that have been used for designing the same, the proposed structure in this paper is a much simpler design and moreover makes use of DM soliton pulses as data signals.

\section{SIMULATION SETUP}

The simulation setup for FULL ADDER is shown in fig.1 was performed using OptSim 5.2 by RSoft a simulation tool for designing advanced optical systems The data streams Data1 at wavelength $11557.75 \mathrm{~nm}$, data2 at wavelength $1557.75 \mathrm{~nm}$ and data3 at wavelength $1557.75 \mathrm{~nm}$ are generated by the mode locked lasers (MLLaser_1, MLLaser_2 \& MLLaser_5 ). The output of the mode locked lasers i.e. soliton pulses are passed through a dispersion management block where they acquire the characteristics of dispersion managed soliton pulses. The dispersion managed soliton are modulated by an electrical PRBS (pseudo random binary sequence) generator. The custom files are used to provide data through PRBS generators. The two data streams data 1 and 
data2 are combined to generate single stream using an optical multiplexer OptMux1 and then through probe given to The setup consists of two XOR gates and two AND gates and one OR gate. Sum is generated using two XOR gates [8] and Carry is generated by applying OR logic to two AND gates [9]. For this purpose, Cross Gain Modulation which produces non-linear effect in SOA cavity is used. Three high power control pulse (pump) and three low power probes are used. These control pulses are shot together with probes into SOA to realize different logic (XOR, AND, OR). For sum output, XOR logic is used and for carry AND and OR logic are used.

For SUM, The probe signal is generated by mode locked laser ML Laser_5 (at wavelength $1550 \mathrm{~nm}$ ) and a custom file of alternating ' 1 ' and ' 0 ' is given from Clock 2 which is given to XOR logic compound component sumhadder, and then data_3 is amplified using EdfaBB1 and the combined data from OptMux2 is amplified by edfa and then through Optcoup1, is given to SOAT1.OptFilt1 is tuned at $1554 \mathrm{~nm}$ wavelength and $0.1 \mathrm{~nm}$ bandwidth to obtain the required spectrum which is inside compound component sumfulladd. carryhadder(contains logic AND).

The resultant SUM output was observed using signal analyzer sumfadder.

For CARRY, the data is combined by OptMux 3 through and 2 and carryhadder compound component, both of the components have Optical filters of wavelength 1490nm used for AND operation. An EDFA amplifier (Erbium Doped Fiber Amplifier) EdfaBB9 is used for amplifying of weak signal. The probe signal of wavelength $1550 \mathrm{~nm}$ is generated using another mode locked laser MLLaser_6 and a custom file of alternating ' 1 ' and ' 0 ' is given from Clock_sequence1. The combined data and probe data is introduced to optical coupler OptCoup2 and then the combined data through OptMux4 is introduced to SOA2(semiconductor optical amplifier). Cross gain modulation (XGM) takes place inside $\mathrm{SOA} 2$ and gain of amplifier is modulated. An optical filter OptFilt2 with centre wavelength $1548.50 \mathrm{~nm}$ (the mean of wavelengths of data1, data2 \& data3) and bandwidth $0.1 \mathrm{~nm}$ was used to select the required spectrum of data streams. The resultant CARRY output was observed using signal analyzer carryfadder.

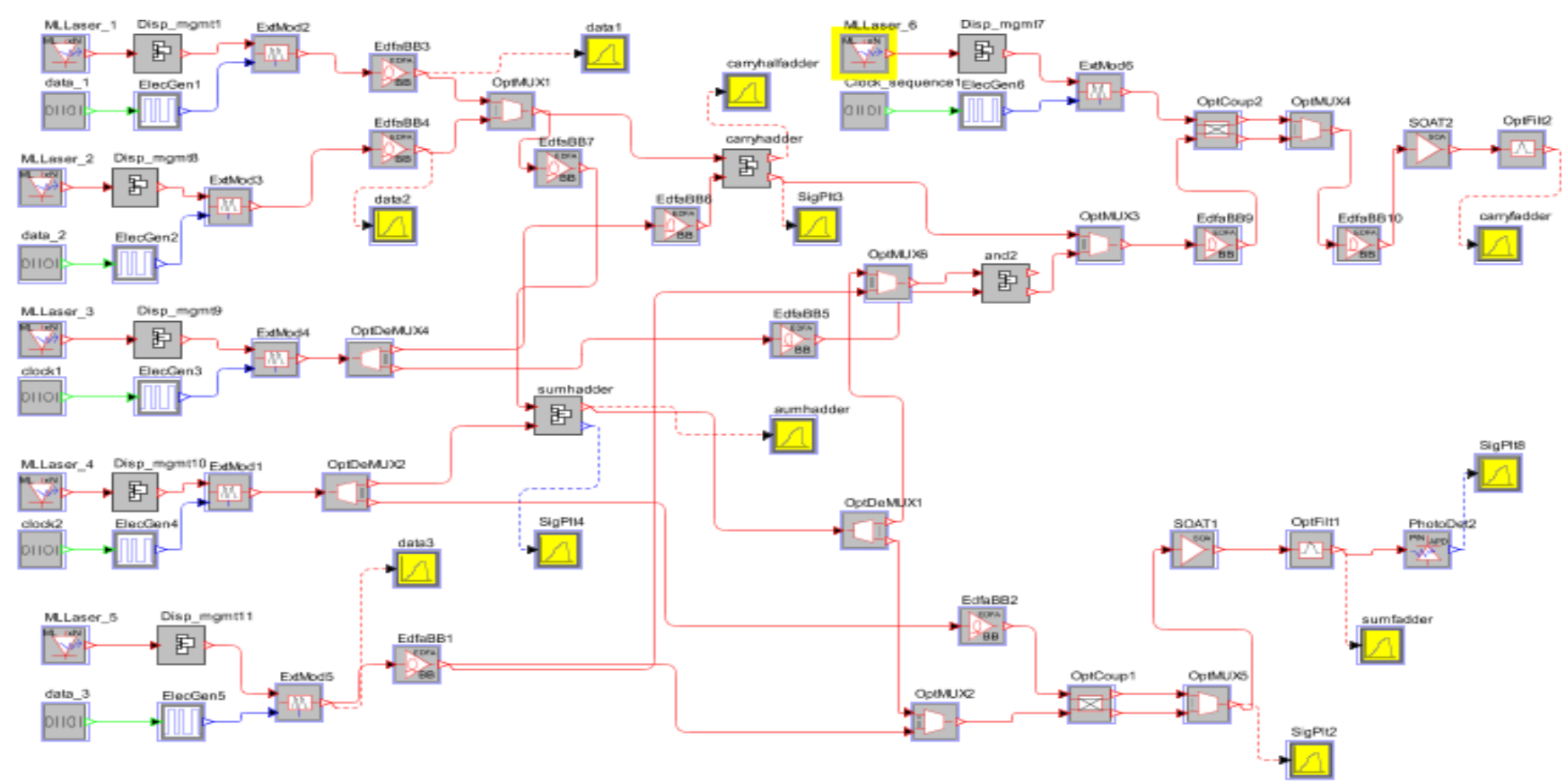

Fig. 1.Simulation setup for All-Optical Full Adder 


\section{RESULTS AND DISCUSSIONS}

The input data streams of data1, data 2 and data 3 are shown in Fig.2, Fig.3 and Fig.4 respectively. As we know that in FULL ADDER, The sum output is high when either one input is high or all the inputs are high and carry output is high only if any of two inputs are high or all inputs are high. In the proposed all-optical full adder, same output was confirmed as the output waveform of resultant signal i.e. sum as shown in fig. 5 and carry as shown in fig.6

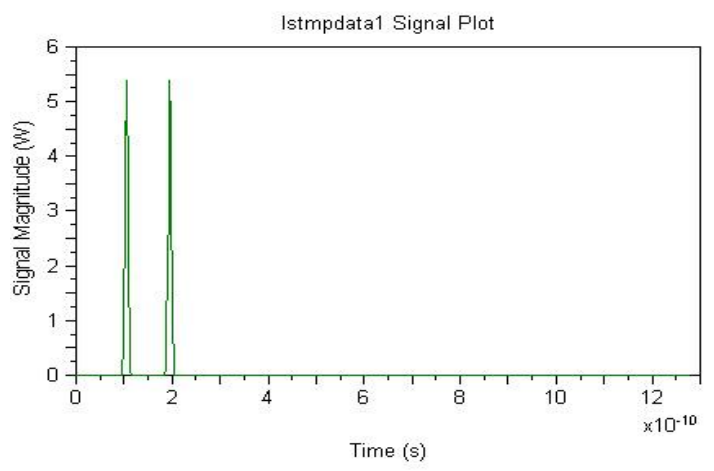

Fig 2.Input data 1(0010001000000)

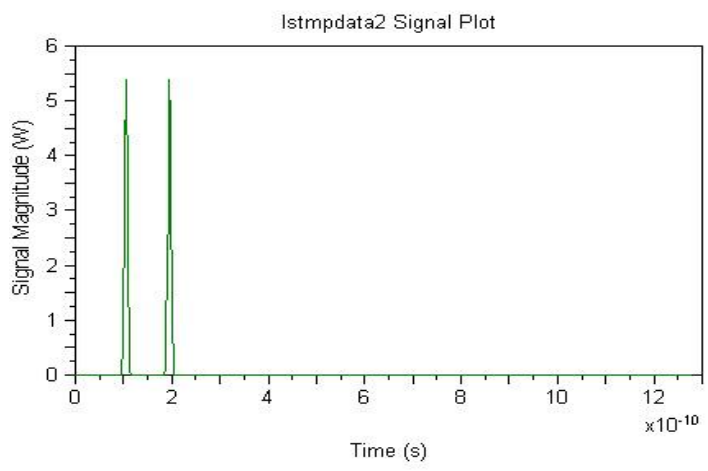

Fig. 3.Input data 2(0010001000000)

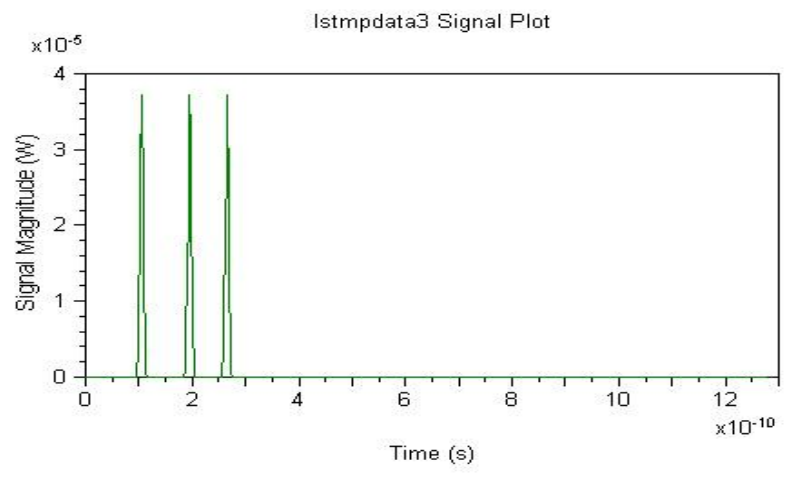

Fig. 4.Input data 3(0010001000100)

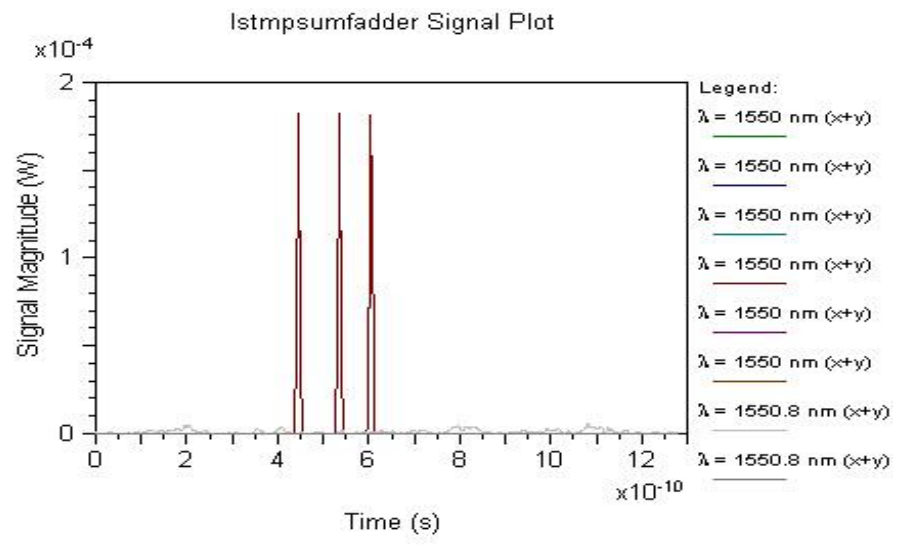

Fig. 5.Ouput signal SUM Full Adder

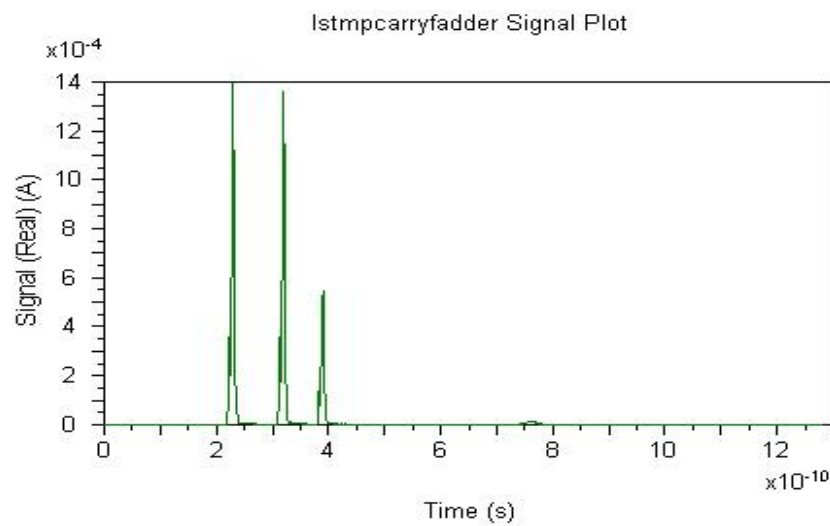

Fig. 6.Ouput signal CARRY Full Adder

Table 1- List of parameters for realizing Full Adder

\begin{tabular}{|c|c|}
\hline Parameter & Value \\
\hline Bit rate & $100 \mathrm{~Gb} / \mathrm{s}$ \\
\hline $\begin{array}{c}\text { Wavelength of Data } \\
\text { 1,Data2,Data3 }\end{array}$ & $1557.75 \mathrm{~nm}$ \\
\hline Wavelength of Probe 1 & $1555.7 \mathrm{~nm}$ \\
\hline Wavelength of Probe 2 & $1548 \mathrm{~nm}$ \\
\hline Wavelength of Probe 2 & $1550 \mathrm{~nm}$ \\
\hline Modulation & Ideal \\
\hline Drive type & On-off ramp \\
\hline Signal type & Voltage \\
\hline Modulation type & NRZ \\
\hline Gain of EDFA & $82 \mathrm{db}$ \\
\hline Psat of EDFA & $18 \mathrm{dbm}$ \\
\hline BW of EDFA & $0.3 \mathrm{~nm}$ \\
\hline
\end{tabular}




\begin{tabular}{|c|c|}
\hline Pump current of SOA2 & $0.30 \mathrm{~A}$ \\
\hline Pump current of SOA1 & $0.35 \mathrm{~A}$ \\
\hline Spectral Shape of SOA & Parabolic \\
SOAs & 5 \\
\hline Confinement factor of SOAs & 0.6 \\
\hline Internal loss of SOAs & $900 \mathrm{~m}^{-1}$ \\
\hline Gain slope of SOAs & $2.78 \mathrm{E}-20 \mathrm{~m}^{-2}$ \\
\hline Length of SOAs & $0.05 \mu \mathrm{m}$ \\
\hline Width of SOAs & $3 \mu \mathrm{m}$ \\
\hline Thickness of SOAs & $0.08 \mu \mathrm{m}$ \\
\hline Central Wavelength of Optfift1 & $1554 \mathrm{~nm}$ \\
\hline Central Wavelength of Optfilt2 & $1548.5 \mathrm{~nm}$ \\
\hline Filter Type & Fabry Parot \\
\hline Bandwidth of filters & $0.1 \mathrm{~nm}$ \\
\hline
\end{tabular}

\section{CONCLUSION}

The all-optical Full Adder was successfully demonstrated by employing DM soliton pulses and XGM behave of semiconductor optical amplifier. Performance of all-optical full adder is verified through simulation. The proposed full adder design has a simpler structure which can be used for photonic integration. The simulated system has the prospective to operate at $100 \mathrm{~Gb} / \mathrm{s}$ and can be used for high speed optical networks, opamp circuits, adaptive filter implementation and in all-optical computing as well in future.

\section{REFERENCES}

[1] Xinliang Zhang, Ying Wang, Junqiang Sun, Deming Liu and Dexiu Huang, 'Novel all-optical AND gate based on cascaded single-port-coupled SOAs'.

[2] Sanmukh Kaur, R.S.Kaler, January 2012, 'All optical integrated full adder-subtractor and demultiplexer using SOA-based Mach-Zehnder interferometer', International
Journal of Engineering Science and Technology (IJEST), ISSN : 0975-5462, Vol. 4 No.01

[3] Q Liu,Z Ouyang and group, 2008. 'All Optical Half adder based on cross structures in two dimensional photonic crystals' Optics Express, Vol.16, Issue23.

[4] Sappasit Thongmeea,* and Preecha P.Yupapinb, 2010, 'All Optical Half Adder/Subtractor using Dark-bright Soliton Conversion Control, 2ndInternational Science, SocialScience, Engineering and Energy Conference: Engineering Science and Management.

[5] Mohamed Nady, Khali F.A. Hussein and Abd-El-hadi A. Ammar, 2013, 'Ultrafast All-Optical Full Adder using Quantum Dot Semiconductor Optical Amplifier Based Mach-Zehnder Interferometer', Progress in Electromagnetic research B, Vol. 54, pp 69-88.

[6] N. J. Smith, W. Forysiak, and N. J. Doran, Jan. 1996, "Reduced Gordon-Haus jitter due to enhanced power solitons in strongly dispersion-managed systems," Electron. Lett., vol. 32, pp. 2085-2086.

[7] John M. Jacob, Ekaterina A. Golovchenko, Alexei N. Pilipetskii, Gary M. Carter, Senior Member, IEEE, and Curtis R. Menyuk, Senior Member, IEEE, October 1997, ' $10-\mathrm{Gb} / \mathrm{s}$ Transmission of NRZ over $10000 \mathrm{~km}$ and solitons over $13,500 \mathrm{~km}$ error free in the same dispersion managed system', IEEE PHOTONICS TECHNOLOGY LETTERS, VOL. 9, NO. 10.

[8] Kadam B. , Gupta N, 2012, 'All Optical XOR Gate Using Single SOA AT $100 \mathrm{~GB} / \mathrm{S}$ ', International Journal of Computational Intelligence Techniques ISSN: 0976-0466 \& E-ISSN: 0976-0474, Volume 3, Issue 2, pp.-86-89.

[9] Kadam Bhambri AND Neena Gupta, 2012.' Implementation of All-Optical AND \& OR Gates using SOA' Journal of Information Systems and Communication ISSN: 0976-8742 \& E-ISSN: 0976-8750, Volume 3, Issue 2, pp.-371-374.

[10] Scaardi, M., P. Ghel, E. Lazzeri, L. Poti, and A Bogoni, Photonic processing for digital comparison and full addition based on semiconductor optical amplifiers," IEEE Journal of Quantum Electronics, Vol. 14, No. 3, pp. 826832. 